\title{
CRITICAL POINTS OF REAL ENTIRE FUNCTIONS AND A CONJECTURE OF PÓLYA
}

\author{
YOUNG-ONE KIM
}

(Communicated by Albert Baernstein II)

To the memory of Professor Jongsik Kim

\begin{abstract}
Let $f(z)$ be a nonconstant real entire function of genus $1^{*}$ and assume that all the zeros of $f(z)$ are distributed in some infinite strip $|\operatorname{Im} z| \leq$ $A, A>0$. It is shown that (1) if $f(z)$ has $2 J$ nonreal zeros in the region $a \leq \operatorname{Re} z \leq b$, and $f^{\prime}(z)$ has $2 J^{\prime}$ nonreal zeros in the same region, and if the points $z=a$ and $z=b$ are located outside the Jensen disks of $f(z)$, then $f^{\prime}(z)$ has exactly $J-J^{\prime}$ critical zeros in the closed interval $[a, b],(2)$ if $f(z)$ is at most of order $\rho, 0<\rho \leq 2$, and minimal type, then for each positive constant $B$ there is a positive integer $n_{1}$ such that for all $n \geq n_{1} f^{(n)}(z)$ has only real zeros in the region $|\operatorname{Re} z| \leq B n^{1 / \rho}$, and (3) if $f(z)$ is of order less than $2 / 3$ then $f(z)$ has just as many critical points as couples of nonreal zeros.
\end{abstract}

\section{INTRODUCTION}

This paper is concerned with the zeros of successive derivatives of real entire functions. An entire function $f(z)$ which assumes only real values on the real axis is said to be a real entire function. In order to state the background to our results, as well as the results themselves, we introduce the following notations: Let $f(z)$ be a nonconstant real entire function. Suppose that $\xi$ is a real zero of $f^{(l)}(z)$ of multiplicity $m$ but not a zero of $f^{(l-1)}(z)$. That is,

$$
f^{(l-1)}(\xi) \neq 0, \quad f^{(l)}(\xi)=f^{(l+1)}(\xi)=\cdots=f^{(l+m-1)}(\xi)=0, \quad f^{(l+m)}(\xi) \neq 0 .
$$

Put

$$
k= \begin{cases}\frac{m}{2}, & \text { if } m \text { is even, } \\ \frac{m+1}{2}, & \text { if } m \text { is odd and } \quad f^{(l-1)}(\xi) f^{(l+m)}(\xi)>0, \\ \frac{m-1}{2}, & \text { if } m \text { is odd and } \quad f^{(l-1)}(\xi) f^{(l+m)}(\xi)<0 .\end{cases}
$$

If $k>0$ we shall say that $\xi$ is a critical zero of $f^{(l)}(z)$ of the multiplicity $k$. The critical zeros of the derivatives of $f(z)$ are called by Fourier the critical points of

Received by the editors March 28, 1994 and, in revised form, September 7, 1994.

1991 Mathematics Subject Classification. Primary 30D15, 30D35.

Key words and phrases. Pólya-Wiman conjecture, Laguerre-Pólya class, Fourier critical point.

This research is supported by the research grant of the Ministry of Education, Republic of Korea, and SNU-GARC. 
$f(z)$. Let $K\left(f^{(l)}\right), l=1,2, \cdots$, be the sum of the multiplicities of the critical zeros of $f^{(l)}(z)$, and let $K_{T}(f)=\sum_{l=1}^{\infty} K\left(f^{(l)}\right) . K\left(f^{(l)}\right)$ is called the number of critical zeros of $f^{(l)}(z)$, and $K_{T}(f)$ is called the total number of critical points of $f(z)$. On the other hand, $Z_{C}(f)$ will denote the number of nonreal zeros of $f(z)$, counting multiplicities. (If $f(z)$ is a constant function, then we set $K\left(f^{(l)}\right)=0, l=1,2, \cdots$, and $Z_{C}(f)=0$.)

If $f(z)$ is a real polynomial, it is easy to see that

$$
Z_{C}(f)-Z_{C}\left(f^{\prime}\right)=2 K\left(f^{\prime}\right)
$$

and

$$
\lim _{n \rightarrow \infty} Z_{C}\left(f^{(n)}\right)=0
$$

Hence, as a result of (1.1) and (1.2), we have

$$
Z_{C}(f)=2 K_{T}(f)
$$

The purpose of this paper is to generalize (1.1), (1.2) and (1.3) to some classes of transcendental functions.

A real entire function $f(z)$ is said to be of genus $1^{*}$ if it can be expressed in the form

$$
f(z)=e^{-\alpha z^{2}} g(z)
$$

where $\alpha \geq 0$ and $g(z)$ is a real entire function of genus at most one. Let $f(z)$ be a nonconstant real entire function and let $\alpha \pm i \beta$, with $\alpha, \beta \in \mathbb{R}$, denote a pair of conjugate nonreal zeros of $f(z)$. The closed disk centered at the point $z=\alpha$ with radius $|\beta|$ is called a Jensen disk of $f(z)$, and the union of the Jensen disks of $f(z)$ will be denoted by $\mathcal{J}(f)$. It is known that if a real entire function $f(z)$ is of genus $1^{*}$, then all the nonreal zeros and critical zeros of $f^{\prime}(z)$ are distributed in the set $\mathcal{J}(f)$. (A proof of this fact will be given in Section 2.)

In 1930, G. Pólya showed that if $f(z)$ is of genus $1^{*}$ and if $Z_{C}(f)<\infty$, then (1.1) is true of $f(z)$ [P1, Theorem I, pp. 25-26]. In the same paper he established the following three "hypothetical theorems."

A. A real integral function of genus 0 has just as many critical points as couples of imaginary zeros.

B. If an integral function of genus $1^{*}$ has only a finite number of imaginary zeros, it has just as many critical points as couples of imaginary zeros.

C. If an integral function of genus $1^{*}$ has only a finite number of imaginary zeros, its derivatives from a certain one onward, let us say $f^{(m)}(z), f^{(m+1)}(z), \cdots$, have no imaginary zeros at all.

From Pólya's result mentioned above, we see that the hypothetical theorems B and $\mathrm{C}$ are equivalent. The hypothetical theorem $\mathrm{C}$ is known as the Pólya-Wiman conjecture and it has been completely proved by T. Craven, G. Csordas, W. Smith 
and the author [CCS1, CCS2, K1, K2], but it seems that no progress has been made on the hypothetical theorem A since 1930. Note that the hypothetical theorem A includes the assertion that the number of critical points and that of nonreal zeros are simultaneously infinite.

It is known that if a real entire function $f(z)$ is not of genus $1^{*}$, then (1.1) and (1.2) are not true in general [HSW, HW1, HW2, L, LO, P2, S]. On the other hand, as the function $f(z)=e^{z}+1$ shows, (1.3) is not true for some functions of genus $1^{*}$. Note that $f(z)=e^{z}+1$ is of order 1 and mean type, and its zero set is $\{(2 n+1) \pi i \mid n=0, \pm 1, \pm 2, \cdots\}$. Hence it is natural to consider real entire functions which are at most of order 1 and minimal type (as in the hypothetical theorem A) or real entire functions of genus $1^{*}$ whose (nonreal) zeros are sufficiently close to the real axis.

In this paper, we will show that if $f(z)$ is a real entire function of genus $1^{*}$, then (1.1) holds for $f(z)$ in each compact connected component of the set $\mathcal{J}(f)$ by

Theorem 1. Let $f(z)$ be a nonconstant real entire function of genus $1^{*}$ and suppose that $a, b$ are real numbers with $a<b$ and $a, b \notin \mathcal{J}(f)$. Then $f(z)$ and $f^{\prime}(z)$ have finitely many zeros in the region $a \leq \operatorname{Re} z \leq b$. Moreover, if $f(z)$ has $2 J$ nonreal zeros in $a \leq \operatorname{Re} z \leq b$ and if $f^{\prime}(z)$ has $2 J^{\prime}$ nonreal zeros in $a \leq \operatorname{Re} z \leq b$, then $f^{\prime}(z)$ has exactly $J-J^{\prime}$ critical zeros in the closed interval $[a, b]$. In particular there are no critical zeros of $f^{\prime}(z)$ outside the set $\mathcal{J}(f)$.

Next, we will prove the following theorem which states that if the real entire function $f(z)$ is at most of order 2 and minimal type, and if the imaginary parts of the zeros of $f(z)$ are uniformly bounded, then (1.2) holds for $f(z)$ locally.

Theorem 2. Let $f(z)$ be a nonconstant real entire function and assume that $f(z)$ is at most of order $\rho, 0<\rho \leq 2$, and minimal type. If there is a positive real number $A$ such that all the zeros of $f(z)$ are distributed in the infinite strip $|\operatorname{Im} z| \leq A$, then for any positive constant $B$ there is a positive integer $n_{1}$ such that $f^{(n)}(z)$ has only real zeros in $|\operatorname{Re} z| \leq B n^{\frac{1}{\rho}}$ for all $n \geq n_{1}$.

Finally, with the aid of Theorem 1 and Theorem 2, we will prove the following limited version of Pólya's hypothetical theorem A.

Theorem 3. Let $f(z)$ be a nonconstant real entire function of order $\rho$, and let $\rho_{C}$ be the convergence exponent of the nonreal zeros of $f(z)$. If there is a positive real number $A$ such that all the zeros of $f(z)$ are distributed in the infinite strip $|\operatorname{Im} z| \leq A$ and if $\rho+2 \rho_{C}<2$, then $Z_{C}(f)=2 K_{T}(f)$.

Since the convergence exponent of the zeros of an entire function $f(z)$ does not exceed the order of $f(z)$, we have the following.

Corollary. Let $f(z)$ be a nonconstant real entire function of order less than $2 / 3$. If there is a positive real number $A$ such that all the zeros of $f(z)$ are distributed in the infinite strip $|\operatorname{Im} z| \leq A$, then $Z_{C}(f)=2 K_{T}(f)$.

The proof of Theorem 1 will be given in Section 2, and the proofs of Theorem 2 and Theorem 3 will be given in Section 3. This paper concludes with some remarks on Theorem 2 and Theorem 3 and some open problems (Section 4). 


\section{Real entire functions of genus $1^{*}$ (the proof of Theorem 1 )}

Let $f(z)$ be a nonconstant real entire function of genus $1^{*}$. Then $f(z)$ can be expressed in the form

$$
f(z)=c z^{n} e^{-\alpha z^{2}+\beta z} \prod_{k}\left(1-\frac{z}{a_{k}}\right) e^{\frac{z}{a_{k}}} \prod_{j}\left(1-\frac{z}{c_{j}}\right)\left(1-\frac{z}{\bar{c}_{j}}\right) e^{\left(\frac{1}{c_{j}}+\frac{1}{\bar{c}_{j}}\right) z}
$$

where $n$ is a nonnegative integer, $\alpha \geq 0, c$ and $\beta$ are real constants, $a_{k}$ are the real zeros of $f(z)$ which are different from 0 , and $c_{j}, \bar{c}_{j}$ are the nonreal zeros of $f(z)$. Of course we have $\sum\left|a_{k}\right|^{-2}<\infty$ and $\sum\left|c_{j}\right|^{-2}<\infty$. Hence the logarithmic derivative of $f(z)$ is given by

$$
\begin{aligned}
\frac{f^{\prime}(z)}{f(z)}= & \frac{n}{z}-2 \alpha z+\beta+\sum_{k}\left(\frac{1}{z-a_{k}}+\frac{1}{a_{k}}\right) \\
& +\sum_{j}\left(\frac{1}{z-c_{j}}+\frac{1}{z-\bar{c}_{j}}+\frac{2 \operatorname{Re} c_{j}}{\left|c_{j}\right|^{2}}\right) .
\end{aligned}
$$

From (2.2), it is easy to see that if $z \notin \mathbb{R}$ and if

$$
z \notin \mathcal{J}(f)=\left\{x+i y \mid\left(x-\operatorname{Re} c_{j}\right)^{2}+y^{2} \leq\left(\operatorname{Im} c_{j}\right)^{2} \text { for some } j\right\},
$$

then

$$
(\operatorname{Im} z)\left(\operatorname{Im} \frac{f^{\prime}(z)}{f(z)}\right)<0 .
$$

In particular, all the nonreal zeros of $f^{\prime}(z)$ are in the set $\mathcal{J}(f)$. This fact was first announced by Jensen, and later proved by Nagy and Walsh.

Jensen's Theorem. Let $f(z)$ be a nonconstant real entire function of genus $1^{*}$ and let $z_{1}$ be a nonreal zero of $f^{\prime}(z)$. Then there is a nonreal zero $z_{0}$ of $f(z)$ such that $\left|z_{1}-\operatorname{Re} z_{0}\right| \leq \operatorname{Im} z_{0}$.

Now suppose that $a<b$ and $a, b \notin \mathcal{J}(f)$. Since all the nonreal zeros of $f(z)$ and $f^{\prime}(z)$ are in the set $\mathcal{J}(f)$, from the definition of the Jensen disks, the absolute values of the imaginary parts of the nonreal zeros of $f(z)$ and $f^{\prime}(z)$ which lie in the region $a \leq \operatorname{Re} z \leq b$ are less than $(b-a) / 2$. Hence $f(z)$ and $f^{\prime}(z)$ have finitely many zeros in the region $a \leq \operatorname{Re} z \leq b$. Assume that $f(z)$ has $2 J$ nonreal and $N$ real zeros, and $f^{\prime}(z)$ has $2 J^{\prime}$ nonreal and $N^{\prime}$ real zeros in the region $a \leq \operatorname{Re} z \leq b$. Moreover we can assume, without loss of generality, that $f(z) f^{\prime}(z) \neq 0$ for $\operatorname{Re} z=a, b$. Let $\Gamma$ be the boundary of the rectangle

$$
R=\left\{x+i y|a \leq x \leq b,| y \mid \leq \frac{b-a}{2}\right\},
$$

and let $\Delta_{\Gamma} \arg \left(f^{\prime}(z) / f(z)\right)$ denote the net change in $\arg \left(f^{\prime}(z) / f(z)\right)$ as the point $z$ traverses $\Gamma$ once over in the counterclockwise direction. Since $\mathcal{J}(f)$ does not intersect $\Gamma,(2.3)$ implies that

$$
\frac{1}{2 \pi} \Delta_{\Gamma} \arg \frac{f^{\prime}(z)}{f(z)}=\frac{1}{2}\left(\operatorname{sgn} \frac{f^{\prime}(a)}{f(a)}-\operatorname{sgn} \frac{f^{\prime}(b)}{f(b)}\right),
$$


where

$$
\operatorname{sgn} r=r /|r|, \quad r \in \mathbb{R} \backslash\{0\} .
$$

In [P1, pp. 29-30], Pólya proved the equation

$$
2 K=N^{\prime}-N-\frac{1}{2}\left(\operatorname{sgn} \frac{f^{\prime}(a)}{f(a)}-\operatorname{sgn} \frac{f^{\prime}(b)}{f(b)}\right),
$$

where $K$ is the number of the critical zeros of $f^{\prime}(z)$ in the closed interval $[a, b]$. Now (2.4) means that

$$
N^{\prime}+2 J^{\prime}=N+2 J+\frac{1}{2}\left(\operatorname{sgn} \frac{f^{\prime}(a)}{f(a)}-\operatorname{sgn} \frac{f^{\prime}(b)}{f(b)}\right)
$$

and hence we have

$$
2 K=N^{\prime}-N-\frac{1}{2}\left(\operatorname{sgn} \frac{f^{\prime}(a)}{f(a)}-\operatorname{sgn} \frac{f^{\prime}(b)}{f(b)}\right)=2\left(J-J^{\prime}\right) .
$$

This proves Theorem 1.

\section{The Class $\mathcal{L P}^{1}$ (The proofs of Theorem 2 And Theorem 3)}

To prove Theorems 2 and 3 , it is enough to consider only those real entire functions whose zeros are distributed in the infinite strip $|\operatorname{Im} z| \leq 1$. Let $\mathcal{L P}^{1}$ denote the class of real entire functions of genus $1^{*}$ all of whose zeros are distributed in the infinite strip $|\operatorname{Im} z| \leq 1$. It is known that $f \in \mathcal{L P} \mathcal{P}^{1}$ if and only if $f(z)$ can be uniformly approximated on compact sets in the plane by a sequence of real polynomials all of whose zeros are distributed in the infinite strip $|\operatorname{Im} z| \leq 1$. (This fact is a consequence of [L, Chapter 8, Theorem 3].) In particular, from the GaussLucas theorem, the class $\mathcal{L} \mathcal{P}^{1}$ is closed under differentiation.

In the proofs of Theorem 2 and Theorem 3, we will use the following.

Lemma 1. Let $\left\{z_{n}\right\}$ be a sequence of complex numbers and assume that $z_{n}=$ $\alpha_{n}+i \beta_{n}, \beta_{n}>0, n=0,1,2, \cdots$. If

$$
\left|z_{n+1}-\alpha_{n}\right| \leq \beta_{n}, \quad n=0,1,2, \cdots
$$

then

$$
\begin{aligned}
& \left|\alpha_{0}-\alpha_{1}\right|+\left|\alpha_{1}-\alpha_{2}\right|+\cdots+\left|\alpha_{n-1}-\alpha_{n}\right| \\
\leq & \sqrt{n\left(\beta_{0}^{2}-\beta_{n}^{2}\right)}, \quad n=1,2, \cdots,
\end{aligned}
$$

and

$$
\begin{gathered}
\quad\left|z_{0}-z_{1}\right|+\left|z_{1}-z_{2}\right|+\cdots+\left|z_{n-1}-z_{n}\right| \\
\leq \beta_{0}-\beta_{n}+\sqrt{n\left(\beta_{0}^{2}-\beta_{n}^{2}\right)}, \quad n=1,2, \cdots .
\end{gathered}
$$

Proof. The first inequality is proved by an induction on $n$, and the second inequality follows from the first one, since $\beta_{n+1} \leq \beta_{n}, n=0,1,2, \cdots$. 
Gontcharoff's First Estimate. Let $f(z)$ be an analytic function in a convex domain D. If $\sup _{z \in \mathcal{D}}|f(z)|=M<\infty$, and if $z, z_{0}, z_{1}, \cdots, z_{n-1} \in \mathcal{D}$, then

$$
\begin{aligned}
& \left|\int_{z_{0}}^{z} \int_{z_{1}}^{\zeta_{1}} \cdots \int_{z_{n-1}}^{\zeta_{n-1}} f\left(\zeta_{n}\right) d \zeta_{n} \cdots d \zeta_{2} d \zeta_{1}\right| \\
\leq & \frac{M}{n !}\left(\left|z-z_{0}\right|+\left|z_{0}-z_{1}\right|+\cdots+\left|z_{n-2}-z_{n-1}\right|\right)^{n} .
\end{aligned}
$$

Proof. See [G, pp. 11-13].

Gontcharoff's Second Estimate. Let $H$ and $\sigma$ be positive real numbers and let $f(z)$ be an entire function such that $M(r ; f)<e^{H r^{\sigma}}$ for all sufficiently large $r$. Let $\alpha$ be an arbitrary positive real number and let $\lambda$ be the positive root of the equation

$$
H \sigma \lambda^{\sigma-1}(\lambda-\alpha)=1 .
$$

Then

$$
\varlimsup_{n \rightarrow \infty} n^{\frac{1}{\sigma}}\left(\frac{M\left(\alpha n^{\frac{1}{\sigma}} ; f^{(n)}\right)}{n !}\right)^{\frac{1}{n}} \leq H \sigma \lambda^{\sigma-1} e^{H \lambda^{\sigma}} .
$$

Proof. See [G, pp. 24-28].

Now we can prove Theorem 2.

Proof of Theorem 2. Let $f(z)$ be a nonconstant real entire function in the class $\mathcal{L P}^{1}$, and suppose that $f(z)$ is at most of order $\rho, \rho \leq 2$, and minimal type. This means that for all $\epsilon>0$ there is a positive real number $r_{1}$ such that

$$
M(r ; f)<e^{\epsilon r^{\rho}},
$$

whenever $r \geq r_{1}$.

Let $n$ be a positive integer, and suppose that $z_{n}$ is a nonreal zero of $f^{(n)}(z)$ which lies in the upper half plane. From Jensen's Theorem, we can find complex numbers $z_{0}, z_{1}, z_{2}, \cdots, z_{n-1}$ such that each $z_{j}, j=0,1, \cdots, n-1$, is a nonreal zero of $f^{(j)}(z)$ and that

$$
\left|z_{j+1}-\operatorname{Re} z_{j}\right| \leq \operatorname{Im} z_{j}, \quad j=0,1, \cdots, n-1 .
$$

Let $z$ be an arbitrary complex number. Since $f^{(j)}\left(z_{j}\right)=0, j=0,1, \cdots, n-1$, we have

$$
f(z)=\int_{z_{0}}^{z} \int_{z_{1}}^{\zeta_{1}} \cdots \int_{z_{n-1}}^{\zeta_{n-1}} f^{(n)}\left(\zeta_{n}\right) d \zeta_{n} \cdots d \zeta_{2} d \zeta_{1}
$$

and hence Gontcharoff's first estimate gives

$$
|f(z)| \leq \frac{M}{n !}\left(\left|z-z_{0}\right|+\left|z_{0}-z_{1}\right|+\cdots+\left|z_{n-2}-z_{n-1}\right|\right)^{n},
$$

where $M$ is the maximum of $\left|f^{(n)}(\zeta)\right|$ on the convex hull of the set $\left\{z, z_{0}, z_{1}, \cdots\right.$, $\left.z_{n-1}\right\}$. 
On the other hand, (3.1) and Lemma 1 give

$$
\left|z_{0}-z_{1}\right|+\left|z_{1}-z_{2}\right|+\cdots+\left|z_{n-1}-z_{n}\right| \leq \operatorname{Im} z_{0}(1+\sqrt{n}) \leq 1+\sqrt{n} .
$$

Hence we get

$$
\left|z_{j}\right| \leq\left|z_{n}\right|+\left|z_{j}-z_{n}\right| \leq\left|z_{n}\right|+1+\sqrt{n}, \quad j=0,1,2, \cdots, n-1,
$$

and

$$
\begin{aligned}
& \left|z-z_{0}\right|+\left|z_{0}-z_{1}\right|+\cdots\left|z_{n-2}-z_{n-1}\right| \\
\leq & |z|+\left|z_{0}\right|+1+\sqrt{n} \\
\leq & |z|+\left|z_{n}\right|+2(1+\sqrt{n}) .
\end{aligned}
$$

Now assume, to get a contradiction, that there is a positive constant $B_{1}$ such that $f^{(n)}(z)$ has a nonreal zero in $|\operatorname{Re} z| \leq B_{1} n^{\frac{1}{\rho}}$ for infinitely many values of $n$. Since $\rho \leq 2$, from (3.2), (3.3) and (3.4), there is a positive constant $B_{2}$ such that

$$
|f(z)| \leq \frac{M\left(B_{2} n^{\frac{1}{\rho}} ; f^{(n)}\right)}{n !}\left(B_{2} n^{\frac{1}{\rho}}\right)^{n}
$$

for $|z| \leq 1$ and for infinitely many values of $n$.

For each $\epsilon>0$ let $\lambda_{\epsilon}$ be the positive real number such that

$$
\epsilon \rho \lambda_{\epsilon}^{\rho-1}\left(\lambda_{\epsilon}-B_{2}\right)=1 .
$$

Since $M(r ; f)=o\left(e^{\epsilon r^{\rho}}\right)$ for all $\epsilon>0$, Gontcharoff's second estimate gives

$$
\varlimsup_{n \rightarrow \infty} B_{2} n^{\frac{1}{\rho}}\left(\frac{M\left(B_{2} n^{\frac{1}{\rho}} ; f^{(n)}\right)}{n !}\right)^{\frac{1}{n}}<B_{2} \epsilon \rho \lambda_{\epsilon}^{\rho-1} e^{\epsilon \lambda_{\epsilon}^{\rho}}, \quad \epsilon>0 .
$$

Now it is easy to see that

$$
\lim _{\epsilon \rightarrow 0} \epsilon \lambda_{\epsilon}^{\rho-1} e^{\epsilon \lambda_{\epsilon}^{\rho}}=0
$$

and hence (3.5) and (3.6) give

$$
f(z)=0, \quad|z| \leq 1
$$

This contradiction shows that our assertion is true.

Let $f(z)$ be a nonconstant real entire function. We will use the notation $\rho(f)$ to denote the order of $f(z)$. On the other hand, the convergence exponent of the nonreal zeros of $f(z)$ and that of the critical zeros of $f^{\prime}(z)$ will be denoted by $\rho_{C}(f)$ and $\rho_{K}\left(f^{\prime}\right)$, respectively. That is

$$
\rho_{C}(f)=\varlimsup_{r \rightarrow \infty} \frac{\log n_{C}(r)}{\log r}, \quad \rho_{K}\left(f^{\prime}\right)=\varlimsup_{r \rightarrow \infty} \frac{\log n_{K}(r)}{\log r},
$$

where $n_{C}(r)$ is the number of the nonreal zeros of $f(z)$ in $|z| \leq r$, and $n_{K}(r)$ is the number of the critical zeros of $f^{\prime}(z)$ in $|z| \leq r$.

In the proof of Theorem 3, we will require the following lemma. 
Lemma 2. If $f(z)$ is a nonconstant real entire function in the class $\mathcal{L P}^{1}$, then

$$
\rho_{C}(f)=\max \left\{\rho_{C}\left(f^{\prime}\right), \rho_{K}\left(f^{\prime}\right)\right\} .
$$

Proof. Let $f(z)$ be a nonconstant real entire function in the class $\mathcal{L} \mathcal{P}^{1}$. Enumerate the real zeros of $f(z)$ as follows:

$$
\begin{aligned}
\cdots \leq a_{k-1} \leq & a_{k} \leq a_{k+1} \leq \cdots \\
& (-\infty \leq \alpha \leq k \leq \omega \leq+\infty, \quad k \text { finite }) .
\end{aligned}
$$

(In this sequence, a real zero of multiplicity $m$ must appear exactly $m$ times.) According to Rolle's theorem, we can find a sequence $\left\{b_{k}\right\}$ of real zeros of $f^{\prime}(z)$ which satisfies

$$
a_{k} \leq b_{k} \leq a_{k+1} \quad \text { for all } k<\omega .
$$

Note that $f^{\prime}(z)$ can have real zeros which do not appear in the sequence $\left\{b_{k}\right\}$. These zeros are called the extra zeros of $f^{\prime}(z)$. It is easy to see that the convergence exponent of the critical zeros of $f^{\prime}(z)$ is equal to that of the extra zeros of $f^{\prime}(z)$.

Now set

$$
\psi(z)=\prod_{\left|a_{k}\right|,\left|b_{k}\right| \leq 1}\left(\frac{z-b_{k}}{z-a_{k}}\right) \prod_{\left|a_{k}\right|,\left|b_{k}\right|>1}\left(\frac{1-z / b_{k}}{1-z / a_{k}}\right) .
$$

(If $f(z)$ has no real zeros at all, set $\psi(z) \equiv 1$, and if it has only one real zero $a_{0}$, set $\psi(z)=\left(z-a_{0}\right)^{-1}$.)

It is well known that the product (3.7) converges uniformly on any compact set not containing the points $a_{k}$, and that the function $w=\psi(z)$ maps the upper half plane into a half plane [L, p.308]. Moreover, since $f \in \mathcal{L P}^{1},(2.3)$ implies that the logarithmic derivative $w=f^{\prime}(z) / f(z)$ maps the half plane $\operatorname{Im} z>1$ into the lower half plane $\operatorname{Im} w<0$. Hence, from Carathéodory's estimate on functions mapping a half plane into a half plane [L, p.18], there are positive constants $c_{1}$ and $c_{2}$ such that

$$
c_{1} r^{-2}<\left|\frac{f^{\prime}(i r)}{f(i r)} \frac{1}{\psi(i r)}\right|<c_{2} r^{2}
$$

for all sufficiently large $r$.

Let $\Pi(z)$ and $\Pi_{1}(z)$ be the canonical products of the nonreal zeros of $f(z)$ and $f^{\prime}(z)$, respectively. The logarithmic derivative of $f(z)$ can be expressed in the form

$$
\frac{f^{\prime}(z)}{f(z)}=\frac{\Pi_{1}(z)}{\Pi(z)} \psi(z) \phi(z)
$$

where $\phi(z)$ is a real entire function. It is clear that $\Pi(z), \Pi_{1}(z)$ and $\phi(z)$ are of genus at most 1 , and the zeros of $\phi(z)$ are exactly the extra zeros of $f^{\prime}(z)$. Moreover, the zeros of $\Pi(z), \Pi_{1}(z)$ and $\phi(z)$ are distributed in the infinite strip $|\operatorname{Im} z| \leq 1$. Therefore, with the aid of (3.8) and (3.9), we obtain the following:

$$
\begin{aligned}
\rho_{C}(f) & =\varlimsup_{r \rightarrow \infty} \frac{\log \log |\Pi(i r)|}{\log r} \\
& =\varlimsup_{r \rightarrow \infty} \frac{\log \log \left|\Pi_{1}(i r) \phi(i r)\right|}{\log r} \\
& =\max \left\{\rho_{C}\left(f^{\prime}\right), \rho_{K}\left(f^{\prime}\right)\right\} .
\end{aligned}
$$


Proof of Theorem 3. Let $f(z)$ be a nonconstant real entire function in the class $\mathcal{L P}^{1}$ and assume that $\rho(f)+2 \rho_{C}(f)<2$. If $Z_{C}(f)<\infty$, then our assertion follows from [CCS1, Theorem 4]. On the other hand, if $Z_{C}(f)=\infty$ and if $Z_{C}\left(f^{(n)}\right)<\infty$ for some $n$, then there is a nonnegative integer $N$ such that $Z_{C}\left(f^{(N)}\right)=\infty$ and $Z_{C}\left(f^{(N+1)}\right)<\infty$. From Lemma $2, \rho_{C}\left(f^{(N)}\right)$ is less than or equal to $\rho_{C}(f)$ which is less than 1. Thus the set $\mathcal{J}\left(f^{(N)}\right)$ is composed of infinitely many compact connected components, and hence Theorem 1 implies that $f^{(N+1)}(z)$ has infinitely many critical zeros.

Now assume that $Z_{C}(f)=Z_{C}\left(f^{(n)}\right)=\infty, n=1,2, \cdots$. From Lemma 2 , the inequality $\rho\left(f^{(n)}\right)+2 \rho_{C}\left(f^{(n)}\right)<2$ holds for all $n=1,2, \cdots$. Hence, by the definition of $K_{T}(f)$, it is sufficient to show that some derivative of $f(z)$ has a critical zero, and to do this, it is enough to prove the existence of a positive real number $B$ and positive integers $J$ and $N$ such that

(a) $f(z)$ has $2 J$ nonreal zeros in the region $|\operatorname{Re} z| \leq B$,

(b) $f^{(N)}(z)$ has only real zeros in the same region, and

(c) $\pm B \notin \mathcal{J}(f) \cup \mathcal{J}\left(f^{\prime}\right) \cup \cdots \cup \mathcal{J}\left(f^{(N-1)}\right)$.

Let $\alpha_{j} \pm i \beta_{j}, j=1,2, \cdots$, be the nonreal zeros of $f(z)$. We can assume, without loss of generality, that $0<\left|\alpha_{1}\right| \leq\left|\alpha_{2}\right| \leq \cdots$.

Take positive real numbers $p$ and $q$ such that

$$
\rho(f)<p, \rho_{C}(f)<q \text { and } p+2 q=2 .
$$

Since $\rho_{C}(f)<q$, we have $\sum\left|\alpha_{j}\right|^{-q}<\infty$, and hence

$$
\varlimsup_{j \rightarrow \infty} \frac{\left|\alpha_{j+1}\right|-\left|\alpha_{j}\right|}{\left|\alpha_{j}\right|^{1-q}}=\infty .
$$

In particular, the inequality

$$
\left|\alpha_{j}\right|+3\left|\alpha_{j}\right|^{1-q}<\left|\alpha_{j+1}\right|
$$

holds for infinitely many values of $j$.

On the other hand, Theorem 2 implies that there is a positive integer $n_{1}$ such that

$$
f^{(n)}(z) \text { has only real zeros in }|\operatorname{Re} z| \leq 2 n^{\frac{1}{p}} \text { for all } n \geq n_{1} .
$$

From (3.11), we can find a positive integer $J$ such that

$$
n_{1}^{\frac{1}{p}}<\left|\alpha_{J}\right| \text { and }\left|\alpha_{J}\right|+3\left|\alpha_{J}\right|^{1-q}<\left|\alpha_{J+1}\right| .
$$

Let $N$ be the least integer not less than $\left|\alpha_{J}\right|^{p}$, that is,

$$
\left|\alpha_{J}\right|^{p} \leq N<\left|\alpha_{J}\right|^{p}+1
$$

From (3.13) and (3.14), we have the following inequalities:

$$
\begin{gathered}
n_{1}<\left|\alpha_{J}\right|^{p} \leq N \\
\left|\alpha_{J}\right|+\sqrt{N} \leq N^{\frac{1}{p}}+\sqrt{N}<2 N^{\frac{1}{p}} \\
2 \sqrt{N}<2\left(\left|\alpha_{J}\right|^{p}+1\right)^{\frac{1}{2}}<3\left|\alpha_{J}\right|^{\frac{p}{2}}=3\left|\alpha_{J}\right|^{1-q}<\left|\alpha_{J+1}\right|-\left|\alpha_{J}\right| .
\end{gathered}
$$


From (3.16) and (3.17), we can find a positive real number $B$ such that

$$
\begin{gathered}
\left|\alpha_{J}\right|+\sqrt{N}<B<\left|\alpha_{J+1}\right|-\sqrt{N}, \text { and } \\
B \leq 2 N^{\frac{1}{p}} .
\end{gathered}
$$

Now (a) and (b) follow from (3.12), (3.15), (3.18) and (3.19). Finally, from Jensen's theorem and Lemma 1, we obtain

$$
\mathcal{J}\left(f^{(n)}\right) \subset \bigcup_{j}\left\{z \mid \alpha_{j}-\sqrt{n+1} \leq \operatorname{Re} z \leq \alpha_{j}+\sqrt{n+1}\right\}, n=0,1,2, \cdots .
$$

Hence (3.18) implies (c) and this completes the proof of Theorem 3.

\section{Some Remarks, EXAmples AND OPEN PROBlems}

Let $f \in \mathcal{L P}^{1}$ and let $r_{n}$ be the minimum of the absolute values of the nonreal zeros of $f^{(n)}(z), n=1,2, \cdots$. (If $f^{(n)}(z)$ has only real zeros, set $r_{n}=\infty$.) Theorem 2 can be restated as follows: If $f(z)$ is of order $\rho, 0<\rho<2$, then

$$
\varliminf_{n \rightarrow \infty} \frac{\log r_{n}}{\log n} \geq \frac{1}{\rho}
$$

and if $f(z)$ of order $\rho, 0<\rho \leq 2$, and minimal type, then

$$
\lim _{n \rightarrow \infty} \frac{r_{n}}{n^{\frac{1}{\rho}}}=\infty
$$

Moreover, if $f(z)$ is of order $\rho, 0<\rho<2$, and mean type, then, using the same method as in the proof of Theorem 2 , we obtain

$$
\lim _{n \rightarrow \infty} \frac{r_{n}}{n^{\frac{1}{\rho}}}>0 .
$$

The following example shows that (4.1), (4.2) and (4.3) are, in some sense, the best possible results.

Example 1. Let $0<\rho<2$ and let

$$
f(z)=\prod_{n=1}^{\infty}\left[\left(1-\frac{z^{2}}{a_{n}}\right)\left(1-\frac{z^{2}}{\bar{a}_{n}}\right)\right]^{2^{n}},
$$

where

$$
a_{n}=4^{\frac{n}{\rho}}+i, \quad n=1,2, \cdots .
$$

It is clear that $f \in \mathcal{L} \mathcal{P}^{1}$, and that $f(z)$ is of order $\rho$ and mean type. If $r_{n}$ denotes the minimum of the absolute values of the nonreal zeros of $f^{(n)}(z), n=1,2, \cdots$, then

$$
\lim _{n \rightarrow \infty} \frac{\log r_{n}}{\log n}=\frac{1}{\rho}
$$

and

$$
\varlimsup_{n \rightarrow \infty} \frac{r_{n}}{n^{\frac{1}{\rho}}} \leq 2^{\frac{1}{\rho}}
$$

However, Theorem 2 gives no information on the growth of the sequence $\left\{r_{n}\right\}$ if $f \in \mathcal{L P}^{1}$ is of order 2 and mean type. Thus we are led to the following problem: 
Problem 1. Let $f \in \mathcal{L} \mathcal{P}^{1}$ be of order 2 and mean type, and let $r_{n}$ be the minimum of the absolute values of the nonreal zeros of $f^{(n)}(z), n=1,2, \cdots$. Describe the growth of the sequence $\left\{r_{n}\right\}$.

Theorem 3 states that if $f \in \mathcal{L P}^{1}$ and if $\rho(f)+2 \rho_{C}(f)<2$, then $Z_{C}(f)=$ $2 K_{T}(f)$. But, there are functions $f(z)$ in the class $\mathcal{L} \mathcal{P}^{1}$ for which $Z_{C}(f)=2 K_{T}(f)$ in spite of $\rho(f)+2 \rho_{C}(f) \geq 2$. In fact, for all real entire functions $f(z)$ of genus $1^{*}$ which have finitely many nonreal zeros the equality $Z_{C}(f)=2 K_{T}(f)$ holds, and the function $f(z)$ in Example 1 has infinitely many critical points. Moreover, we have the following example:

Example 2. Consider

$$
f(z)=e^{\beta z} \sum_{m, n=0}^{M} c_{m, n} \cos ^{m} z \sin ^{n} z
$$

where $\beta$ and $c_{m, n}$ are real numbers. Since $e^{-\beta z} f(z)$ is a polynomial of $e^{i z}$ and $e^{-i z}$, it is $2 \pi$ periodic and has only a finite number of zeros in $0 \leq \operatorname{Re} z<2 \pi$. In particular, there is a positive real number $A$ such that all the zeros of $f(z)$ are distributed in the infinite strip $|\operatorname{Im} z| \leq A$. Assume that $f(z)$ has $2 J$ nonreal and $N$ real zeros, and $f^{\prime}(z)$ has $2 J^{\prime}$ nonreal and $N^{\prime}$ real zeros in the region $0 \leq \operatorname{Re} z<2 \pi$. Then we have $N+2 J=N^{\prime}+2 J^{\prime}$. Since $e^{-\beta z} f(z)$ and $e^{-\beta z} f^{\prime}(z)$ are $2 \pi$ periodic functions, $f^{\prime}(z)$ has exactly $J-J^{\prime}$ critical zeros in each interval $[2 l \pi, 2 l \pi+2 \pi)$, $l \in \mathbb{Z}$. On the other hand, Theorem 2 implies that $f^{(k)}(z)$ has only real zeros in $|\operatorname{Re} z| \leq 2 \pi$ for all sufficiently large $k$. Since $e^{-\beta z} f^{(k)}(z)$ is $2 \pi$ periodic for all $k=1,2, \cdots, f^{(k)}(z)$ has only real zeros for all sufficiently large $k$, and hence $f(z)$ has exactly $J$ critical points in each interval $[2 l \pi, 2 l \pi+2 \pi), l \in \mathbb{Z}$.

Now our second problem is as follows:

Problem 2. Characterize those functions $f(z)$ in the class $\mathcal{L P}^{1}$ which have just as many critical points as couples of nonreal zeros.

Finally we emphasize that our argument is not applicable to Pólya's hypothetical theorem A, because it deals with only those functions $f(z)$ for which the imaginary parts of the zeros of $f(z)$ are uniformly bounded.

\section{REFERENCES}

[CCS1] T. Craven, G. Csordas, and W. Smith, The zeros of derivatives of entire functions and the Pólya-Wiman conjecture, Ann. of Math. (2) 125 (1987), 405-431. MR 88a:30007

[CCS2] Z Z Zeros of derivatives of entire functions, Proc. Amer. Math. Soc. 101 (1987), 323-326. MR 88k:30024

[G] W. Gontcharoff, Recherches sur les dérivées successives des fonctions analytiques, Ann. École Norm. 47 (1930), 1-78.

[HSW] S. Hellerstein, L. C. Shen, and J. Williamson, Reality of the zeros of an entire function and its derivatives, Trans. Amer. Math. Soc. 275 (1983), 319-331. MR 84a:30050

[HW1] S. Hellerstein and J. Williamson, Derivatives of entire functions and a question of Pólya, Trans. Amer. Math. Soc. 227 (1977), 227-249. MR 55:8353

[HW2] - Derivatives of entire functions and a question of Pólya. II, Trans. Amer. Math. Soc. 234 (1977), 497-503. MR 58:1151

[K1] Y. O. Kim, A proof of the Pólya-Wiman conjecture, Proc. Amer. Math. Soc. 109 (1990), 1045-1052. MR 90k:30049 
[K2] - On a theorem of Craven, Csordas and Smith, Complex Variables 22 (1993), 207-209. MR 94m:30052

[L] B. Ja. Levin, Distribution of Zeros of Entire Functions, Transl. Math. Monographs, vol. 5, Amer. Math. Soc., Providence, RI, 1964. MR 28:217

[LO] B. Ja. Levin and I. V. Ostrovskii, On the dependence of the growth of an entire function on the distribution of the zeros of its derivatives, Amer. Math. Soc. Transl. Ser. 2, vol. 32, Amer. Math. Soc., Providence, RI, 1963, pp. 323-357. MR 24:A833

[P1] G. Pólya, Some problems connected with Fourier's work on transcendental equations, Quart. J. Math. Oxford Ser. 1 (1930), 21-34.

[P2] - On the zeros of derivatives of a function and its analytic character, Bull. Amer. Math. Soc. 49 (1943), 178-191. MR 4:192d

[S] T. Sheil-Small, On the zeros of the derivatives of real entire functions and Wiman's conjecture, Ann. of Math. (2) 129 (1989), 179-193. MR 90a:30084

Department of Mathematics, College of Natural Sciences, Sejong University, Seoul 133-747, KOREA 